\title{
Conceptual Design of a 2-DoF Planar High-Speed Industrial Parallel Manipulator
}

\author{
Emre Uzunoğlu ${ }^{1}$, Merve Özkahya ${ }^{1}$, Erkan Paksoy $^{1}$, Barış Taner $^{2}$, \\ M. İ. Can Dede ${ }^{1[0000-0001-6220-6678]}$, Gökhan Kiper ${ }^{10000-0001-8793-724 X]}$ \\ ${ }^{1}$ İzmir Institute of Technology, Mechanical Engineering, Turkey \\ ${ }^{2}$ University of Texas at Arlington, Mechanical \& Aerospace Engineering, USA \\ gokhankiper@iyte.edu.tr
}

\begin{abstract}
This study focuses on conceptual design alternatives for a planar high-speed/high-precision manipulator in terms of mechanism structure, control strategy, and drive system selection. These concepts are investigated specifically for planar 5-bar based parallel linkages. An over-constrained 6-bar linkage with parallelogram loops and its simply constrained version are selected for detailed design. In addition, a model-based control strategy including a stiffness model is discussed for future studies. Alternative drive systems are evaluated. Finally a prototype is presented.
\end{abstract}

Keywords: Planar Parallel Manipulator, Conceptual Design, Control Strategy.

\section{Introduction}

Serial robots cannot provide the necessary performance during operations which require high acceleration and precise motion due to the flexibility of the manipulator; therefore manipulators with parallel kinematic structure are preferred in such cases. Although parallel manipulators can be optimally designed according to several performance criteria, the positioning accuracy is still limited in application due to factors such as manufacturing errors, flexibility of links and joints, and joint clearances. This study has emerged from problems encountered during previous studies on enhancing the positioning accuracy of a high-acceleration manipulator [1,2]. The aim of this paper is to investigate structural and control based improvements in order to increase the positioning accuracy of planar parallel robots commonly used in industrial applications. The conceptual design alternatives are presented in terms of mechanism structure, controller strategy, and selection of drive-system components to be used for the design and prototype of a simply constrained and an over-constrained parallel manipulator.

First, a brief literature survey on calibration, balancing, and stiffness analysis are presented in this section. The highlights of these surveys are presented especially on high-speed parallel manipulators and 5-bar based mechanisms. Then, the conceptual 
design alternatives for the mechanism structure, control strategy and drive system are presented and discussed thoroughly. Finally, constructed prototypes are presented.

\subsection{Balancing}

Balancing is a common problem for high-speed machinery. If the potential energy of the system is stationary at all times when the system is under the effect of conservative forces, the system is said to be statically balanced [3]. When resultant of reaction forces and moments of the system due to inertial forces and moments are zero, the system is said to be dynamically balanced. If reaction forces are zero, the system is shaking force balanced and if reaction moments are zero, the system is shaking moment balanced [4]. A dynamically balanced system is already statically balanced.

In the literature, there are several balancing techniques, which are categorized as counter-mass balancing, spring balancing, using duplicate mechanisms, active balancing, designing the mechanism as inherently balanced, etc. In [5] and [6], some counter-mass balancing methods are investigated in detail for force and moment balancing of planar and spatial mechanisms such as full force balancing, full force and partial moment balancing, etc. Shaking forces and moments are significant problems in highspeed machinery. Van der Wijk, Herder and Demeulenaere [7] have compared and evaluated existing complete balancing principles regarding the addition of mass and the addition of inertia in order to compare the balancing performance with added mass and inertia. Complete dynamic balancing for some planar 4R (R: revolute joint), 5R, 3-RRR and spatial 3-RRS parallel mechanisms are presented in [8].

\subsection{Calibration with Stiffness Model}

Manipulator calibration is a cost-effective way to improve robot accuracy, and many researchers have devoted efforts to this field. Different models, measurement systems, and algorithms for identification and compensation have been developed as summarized in [9-11].

For a parallel mechanism, the global platform pose depends on all of its actuators. For our application, the global error at the end of the tool is the main concern. Some part of this error could be due to manufacturing tolerances or assembly. At high speeds, control speed and accuracy play an important role in the overall final position.

Calibration of parallel manipulators requires more complex algorithms, models and measurement methods as compared to serial manipulators. In general, the calibration methods of parallel mechanisms can be categorized as parametrical (or model-based) and numerical methods. Parametrical methods depend on the used sensors and the approach used in calibration [12]. Based on the error model, it is possible to map the geometric errors to the end-effector error of a parallel mechanism. This mapping can be used to increase the global accuracy of the structure [13].

In general, the performance of a robot manipulator is evaluated by two noteworthy points: payload capacity and positional accuracy. Metric for payload capacity is defined as payload/weight ratio in [14]. In evaluation of the positioning accuracy, usually three metrics are used as absolute accuracy, repeatability and resolution [15]. Abso- 
lute accuracy is defined as robot's ability of positioning its end-effector at a desired configuration within its workspace and it is half of the resolution.

[16] presents an off-line compensation method depending on the stiffness properties calculated under the auxiliary loads and external forces. Robot control program that determines the trajectory is modified according to the calculated deformations. Online compensation provides best solution for the compensation of errors due to compliance of robot mechanism. The difficulty in applying an on-line compensation method stems from the complexity of the mathematical stiffness model for especially multi-degree-of-freedom $(\mathrm{DoF})$ systems. Calculation of the stiffness matrix provides the relation between the forces acting on the robot and the compliant displacements of the robot mechanism, which are the reason of the compliant errors at the end-effector.

\section{Conceptual Design}

In order to observe the effect of over-constraint, simply and over-constrained mechanisms with similar structure are to be built and tested. In this Section, first, planar two-DoF mechanism alternatives are presented. Then, the controller strategy and drive system alternatives are discussed.

\subsection{Kinematic Structure Alternatives for a Two-DoF Planar Linkage}

In this Section, two-DoF planar mechanism structure alternatives are investigated. Single loop mechanisms with five links and five single-DoF (revolute (R) and prismatic (P)) joints are considered. More than two P joints cannot be used in a loop, because otherwise there will be internal mobility. In this way, although 32 different mechanisms can be enumerated (with combinations of $0 \mathrm{P}, 1 \mathrm{P}$ and $2 \mathrm{P}$ ), it is possible to obtain 6 different 5-link mechanisms under the following conditions:

1. Actuators must be supported from the base and no actuated joints must carry the load of the other, i.e. the RPRRR is not suitable (underlined letters indicate actuated joints).

2. If available, the $P$ joints must be active.

3. Mirror image mechanism structures are equivalent (ex. RRRPR $=R P R R R)$.

Five-link mechanisms that satisfy these requirements are $\underline{R} R R R \underline{R}, \underline{R} R R \underline{P} R$, $\underline{R} R R R \underline{P}, R \underline{P} R R \underline{P}, R \underline{P} R \underline{P} R$ and $\underline{P R R R} \underline{P}$ [17]. Alıc1 [18] has developed a method for determining the singularity positions of all these six types of mechanisms. Symmetrical structures are generally preferred because of the workspace symmetry, balancing and control simplicity [19]. The mechanisms for which the $\mathrm{P}$ joints are carried by the fixed revolute joints are not favorable due to high inertia of the actuated $\mathrm{P}$ joints. The six mechanisms mentioned just above are evaluated according to the structural symmetry, whether actuators are fixed or not and whether the actuators are of the same type ( $\mathrm{R}$ or $\mathrm{P}$ ) for ease of control. The evaluation is summarized in Table 1 and accordingly $\underline{R} R R R \underline{R}(5 R)$ and $\underline{P R R R} \underline{P}$ alternatives are selected for final evaluation. 
Table 1. Evaluation of five-link mechanisms considering various aspects.

\begin{tabular}{|c|c|c|c|}
\hline Mechanism & Fixed Actuators & Structural Symmetry & Ease of control \\
\hline$\underline{R} R R R \underline{R}$ & yes & yes & yes \\
\hline$\underline{R} R R \underline{P}$ & no & no & no \\
\hline$\underline{\text { RRRRP }} \underline{\text { nos }}$ & yes & no & no \\
\hline RRRR $\underline{P}$ & no & no & no \\
\hline R $\underline{P} R \underline{P}$ & no & yes & no \\
\hline$\underline{P} R R R \underline{P}$ & yes & yes & yes \\
\hline
\end{tabular}

Sun, Cheung, and Lou [19] compared 5R and PRRR $\underline{P}$ mechanisms, which were optimized for the same effective square workspace, with respect to functionality, compactness and the singular values of the forward and inverse Jacobian matrices. It is concluded that a smaller size of the $5 \mathrm{R}$ mechanism can work in the same workspace. The 5R mechanism features lighter weight structure, better precision, resistance, and force transmission capabilities. These advantages reduce actuator power requirements and are suitable for high acceleration applications. The PRRR $\underline{P}$ mechanism, on the other hand, possesses higher velocity influence coefficient for the endeffector and is more suitable for pick-and-place applications.

5R Mechanisms: The generic 5R mechanism (Fig. 1a) is actuated at $A_{0}$ and $B_{0}$ fixed revolute joints. Input variables are $\theta_{1}$ and $\theta_{2}$ angles, whereas $\theta_{3}$ and $\theta_{4}$ are passive joint variables. Point " $E$ " represents the end-effector point. $\left|\mathrm{A}_{0} \mathrm{~A}\right|$ and $|\mathrm{AE}|$ are respectively equal to $\left|\mathrm{B}_{0} \mathrm{~B}\right|$ and $|\mathrm{BE}|$ for a symmetric workspace. If the mechanism is evaluated in terms of construction, there is no problem about fixed revolute joint connections. However, it is difficult to assemble the tool to be used as the end-effector to point "E". Furthermore, the end-effector orientation is uncontrolled unless timing belt-pulley systems are used.

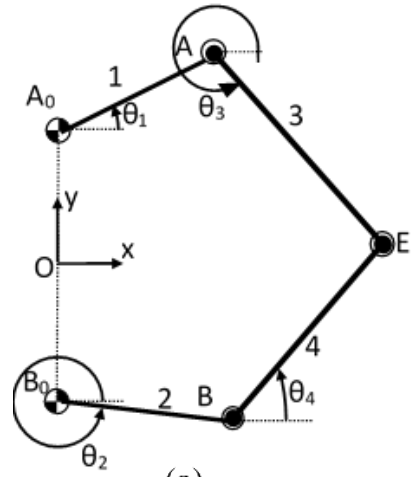

(a)

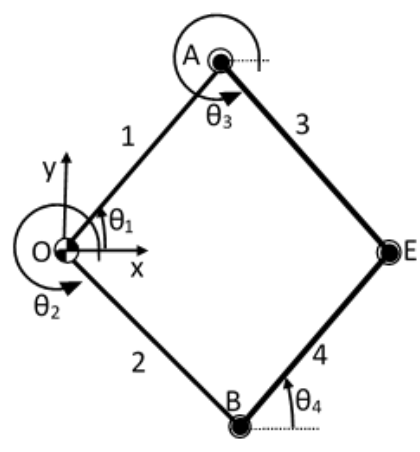

(b)

Fig. 1. (a) General $5 R$ mechanism, (b) $5 R$ mechanism with coincident fixed axes

Fixed revolute joints are coincident at the point "O" in Fig. 2b. All link lengths can be selected as equal to each other for ease of balancing. Although this special case has 
simpler kinematic structure than the general case, the motor connection construction is difficult due to the coincident fixed revolute joint axes. Coincident motor axes can be avoided by using suitable gear connections.

6R Mechanisms with Parallelogram Loops: The construction of base and endeffector for $6 \mathrm{R}$ mechanisms is simpler compared to $5 \mathrm{R}$ mechanisms. There are no coincident axes in this case. A 6R loop is obtained by introducing equal offset between coincident axes and the $\mathrm{E}$ joint of the $5 \mathrm{R}$ mechanism shown in Fig. 1b. However, parallelogram loops should be used for at least one of the arms so that the endeffector orientation is constant. The other option is to use timing belt-pulley systems within the arms. But the belt tension is a problem. If the belt is over-tensioned, excessive joint forces are observed. On the other hand, if the belt tension is not enough, there will be vibrations.

In order to obtain a simply constrained mechanism, two or three parallelogram loops must be used. The simply constrained mechanism with 2 parallelogram loops is shown in Fig. 2a. For symmetric mass distribution, parallelogram loops can be used for both arms, but in this case, the mechanism becomes an over-constrained one. A pin-in-slot (joint "L" in Fig. 2b) can be used instead of one of the passive revolute joints to have a simply constrained mechanism. The mechanism is over-constrained when only $\mathrm{R}$ joints are used for all four parallelogram loops (Fig. 2c).

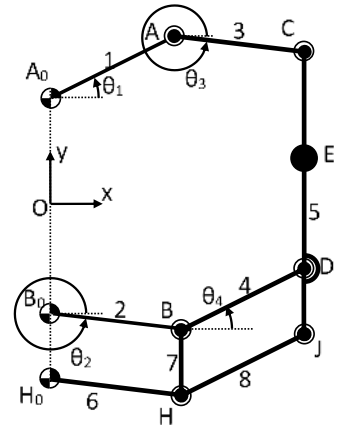

(a)

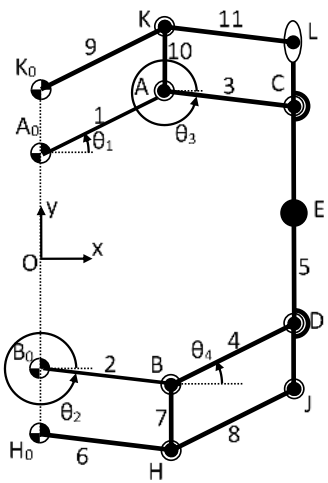

(b)

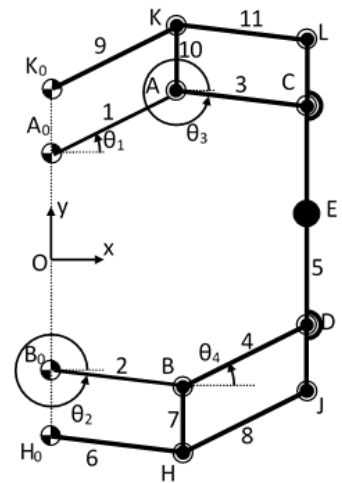

(c)

Fig. 2. (a) Simply constrained $6 \mathrm{R}$ mechanism with 2 parallelogram loops, (b) simply constrained 6R mechanism with pin-in-slot joint, (c) over-constrained 6R mechanism

The over-constrained $6 \mathrm{R}$ mechanism with four parallelogram loops is selected for investigation in this study. For comparison, another equivalent simply-constrained mechanism has to be selected and the following criteria are taken into account to select this mechanism:

1. Ease of balancing and simple mathematical model

2. To have similar construction as the over-constrained mechanism

3. Structural simplicity 
4. Constant end-effector orientation

5. Structural symmetry

6. Kinematic equivalence

The general $5 \mathrm{R}$ mechanism has disadvantage about criterion (1). 5R mechanism with coincident fixed axes is advantageous in terms of criteria (1), (5), (6), but it involves complexity about assembly of motors and the end-effector in terms of criteria (3) and (4). Although the 6R mechanism with two or three parallelogram loops (Fig. 2a) is disadvantageous in terms of criterion (5), it is possible to come-up with a dynamically balanced design. Moreover, it has more advantages than other alternatives in terms of criterion (2). Therefore, the simply constrained 6R mechanism with three parallelogram loops is selected for further investigation. One link of a parallelogram loop is removed from the over-constrained mechanism in order to obtain the simply constrained one.

\subsection{Control Scheme Alternatives}

As discussed in Section 1.2, a better control of a high precision parallel mechanism can be achieved by integrating the stiffness model into the controller. Two conceptual design alternatives have been devised for the control system's flowchart (Fig. 3).

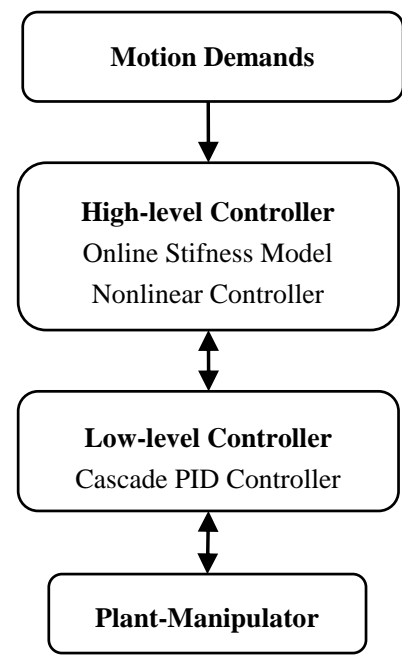

(a)

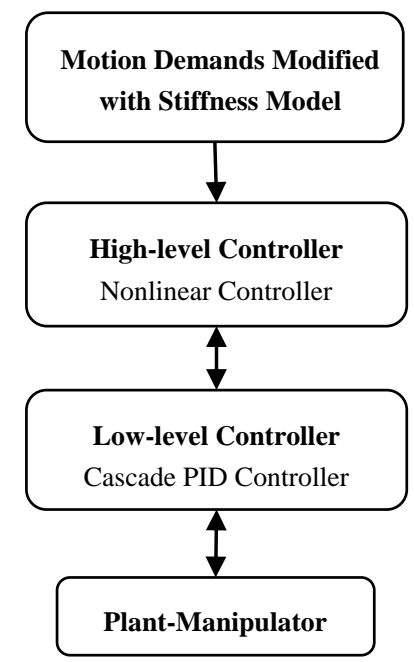

(b)

Fig. 3. Control strategy alternatives

In the first flowchart (Fig. 3a), the trajectory created for end-effector's motion demands are fed to the high-level controller. The high-level controller runs in a computer along with data acquisition device hardware, and thus, the algorithms run in a realtime operating system. The high-level controller algorithm consists of a non-linear 
controller and an online stiffness model. A non-linear controller such as computed torque method or adaptive controller are tested and evaluated in simulation environment to be used in the high-level controller. However, in this scenario, the critical point is the computation time of the stiffness model, which is supposed to run in realtime at a designated frequency of $1 \mathrm{kHz}$.

In the second alternative (Fig. 3b), the end-effector trajectory is modified with a correction algorithm with stiffness model during the pre-process, which is an offline method. The working principle of the stiffness model in this scenario is to calibrate the pre-determined end-point trajectory by estimating the positioning errors due to elastic deformations of the mechanism components. In both alternatives, the low-level controller, which is embedded in motor drivers, is utilized with cascade PID controller and position feedbacks from the motors are sent back to the high-level controller.

\subsection{Drive System Alternatives}

In order to meet the torque and speed requirements for a high-speed industrial application, drive system selections are carried out among available alternatives of industrial servo drives and drivers. AC servo motors are favored due to the high torque per weight values, their efficiency and their frequent use in industrial systems.

Due to the high-speed and high-precision requirements in line with the other conceptual alternatives, the actuation system must be selected in such a way that it can provide high dynamic motion. Accordingly, two actuation system alternatives are evaluated: 1) a direct-drive servo motor, 2) an AC servo motor with a low-backlash $(<1 \mathrm{arcmin})$ reducer that has suitable gear-ratio and efficiency.

Table 2. Drive system alternatives

\begin{tabular}{|c|c|c|}
\hline Criteria & Cycloid Gear + Servo Motor & Direct Drive Servo Motor \\
\hline Efficiency & + & ++ \\
\hline Cycle time & + & ++ \\
\hline Torque-speed range & + & ++ \\
\hline Clearance & + & ++ \\
\hline Wight to power ratio & + & -- \\
\hline Size & + & - \\
\hline Ease of integration & ++ & - \\
\hline \multicolumn{2}{|c|}{ Price } & - \\
\hline
\end{tabular}

Direct drive systems are more efficient systems because there are no efficiency losses and backlashes due to the reducer. However, they are generally disadvantageous in terms of weight and size compared to geared motors. Also, precision equipment is required during the mounting process. In most applications, application-specific frameless motors are used and they require more complex constructional details. A geared motor is disadvantageous compared to the direct drive because of the degradation in terms of dynamic motion and efficiency. However, special low-backlash gearboxes such as harmonic drives and cycloid reducers prevail as relatively better solutions. Finally, complying with the torque and speed requirements of the mechanism, 
two alternatives are compared, which are: Kollmorgen DDR series direct drive motor and AKM series motor with Sumitomo fine cyclo gearbox. The evaluation table based on the technical specifications of the selected drives are provided in Table 2.

\section{Conclusions}

In this paper, the alternatives for mechanism structure, control strategy, and drive system are presented for a high-speed planar robotic manipulator. First, overconstrained and simply constrained mechanism alternatives are presented and evaluated. As a result, a simply-constrained mechanism with three parallelogram loops and an over-constrained 6R mechanism with 4 parallelogram loops are chosen.

Two concepts are introduced to improve the performance of controller by integrating the stiffness model. It is decided that both algorithms can be tested in future studies and these alternatives can be compared with respect to their computational cost and efficiency.

Although a direct drive system is advantageous in terms of efficiency, the system should be as light as possible and its dimensions should be within certain limits. Therefore, a motor with cycloid gear reducer is selected since it also meets the requirements of low backlash. According to alternatives presented here, a prototype for the robotic manipulator is manufactured and assembled as shown in Fig. 4. The link that is removed in Fig. 4b is replaced with two lumped masses at the associated joints in order to obtain dynamic equivalence of the two mechanisms.

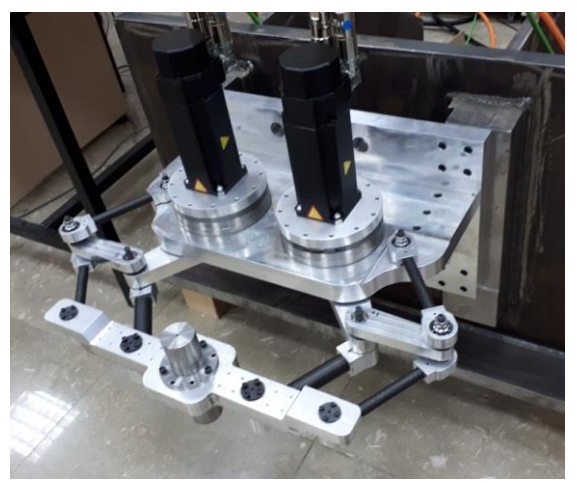

(a)

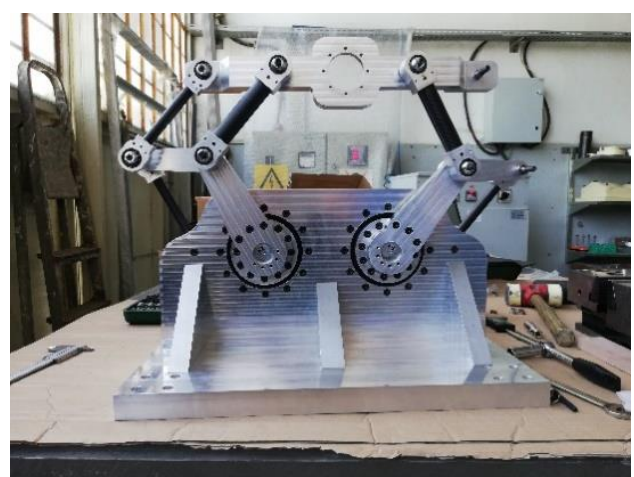

(b)

Fig. 4. Mechanism prototype in (a) over-constrained and (b) simply constrained configuration

Future studies include calibration, tests and design iterations. The tests will be done for both simply and over-constrained mechanisms to see the effect of over-constraint. Both mechanisms will be tested for balanced, partially force-balanced and fully forcebalanced cases. The base vibrations will be monitored with accelerometers. Control strategies including online and off-line stiffness models will be tested. The accuracy and repeatability of the end-effector will be measured using a laser cutter end- 
effector. In the end, the generated know-how will be shared with the academic and industrial community.

\section{Acknowledgment}

This work is financially supported by The Scientific and Technological Research Council of Turkey via grant number 116M272.

\section{References}

1. Uzunoğlu, E., Dede, M. İ. C., Kiper, G.: Trajectory planning for a planar macro-micro manipulator of a laser-cutting machine. Industrial Robot: An International Journal, 43(5), 513-523 (2016).

2. Dede, M. İ. C., Kiper, G., Uzunoğlu, E.: A macro-micro mechanism design for laser cutting process. In: Proc. The 17th International Conference on Machine Design and Production, Bursa, (2016).

3. Jean, M., Gosselin, C. M.: Static balancing of planar parallel manipulators. In: Proc. 1996 IEEE International Conference on Robotics and Automation, Vol.4, pp. $3732-3737$ (1996).

4. Van der Wijk, V.: 'Methodology for Analysis and Synthesis of Inherently Force and Moment-Balanced Mechanisms. PhD Thesis, University of Twente (2014).

5. Lowen, G. G., Berkof, R. S.: Survey of investigations into the balancing of linkages. Journal of Mechanisms, 3(4), 221-231 (1968).

6. Lowen, G. G., Tepper, F. R., Berkof, R. S.: Balancing of linkages-an update. Mechanism and Machine Theory, 18(3), 213-220 (1983).

7. Van der Wijk, V., Herder, J. L., Demeulenaere, B.: Comparison of various dynamic balancing principles regarding additional mass and additional inertia, Journal of Mechanism and Robotics, 1, no 041006 (2009).

8. Van der Wijk, V., Herder, J. L.: Synthesis of dynamically balanced mechanisms by using counter-rotary countermass balanced double pendula. Journal of Mechanical Design, 131, no 111003 (2011).

9. Roth, Z. S., Mooring, B. W., Ravani, B.: An overview of robot calibration. In: IEEE Southcon Conference, pp. 377-384, Orlando, Florida (1986).

10. Hollerbach, J. M.: A survey of kinematic calibration. In: Khatib, O., Craig, K. J., LozanoPérez, T. (eds.) The Robotics Reviews 1, pp. 207-242, MIT Press, Cambridge, MA (1988).

11. Mooring, B.: Fundamentals of Manipulator Calibration. John Wiley and Sons, New York (1991).

12. Hernández-Martínez, E., López-Cajún, C., Jáuregui-Correa, J.: Calibration of parallel manipulators and their application to machine tools. Ingeniería. Investigación y Tecnología, 11, 141-154 (2010).

13. Elatta, A. Y., Li, P. G., Fan, L. Z., Yu, D., Luo, F.: Information Technology Journal, 3(1), 74-78 (2004)

14. Merlet, J. P.: Parallel Robots. Springer, Dordrecht (2006).

15. Klimchik, A.: Enhanced Stiffness Modeling of Serial and Parallel Manipulators for Robotic-Based Processing of High Performance Materials. PhD Thesis, Ecole Centrale de Nantes (2011). 
16. Klimchik, A., Bondarenko, D., Pashkevich, A., Briot, S., Furet, B.: Compliance error compensation in robotic-based milling. In: Informatics in Control, Automation and Robotics, pp. 197-216, Springer (2014).

17. Cervantes-Sánchez, J. J., Rendón-Sánchez, J. G.: A simplified approach for obtaining the workspace of a class of 2-dof planar parallel manipulators. Mechanism and Machine Theory, 34, 1057-1073 (1999).

18. Alıc1, G.: Determination of singularity contours for five-bar planar paralel manipulators. Robotica, 18, 569-575 (2000).

19. Sun, S., Cheung, J. W. F., Lou, Y.: A study on five-bar manipulators for semiconductor packaging applications. In: Proc. 2007 IEEE International Conference on Mechatronics and Automation, pp. 1811-1816 (2007). 